\title{
Zur Position der Bulbi olfactorii bei Knochenfischen unter besonderer Berücksichtigung von Mugil auratus (Actinopterygii, Mugiliformes) $^{1}$
}

\author{
ARTHUR Holl \\ 1. Zoologisches Institut der Justus Liebig-Universität, Gießen
}

\begin{abstract}
On the position of the bulbi olfactorii in bony fishes with special reference to Mugil auratus (Actinopterygii, Mugiliformes). The position of the olfactory bulbs in Mugil auratus Russo resembles that of the Salmonide-type. However, the bulbs are distinctly displaced from the corpora striata. This displacement is caused by a growth in length of the secondary olfactory tracts, which seems to start at a later stage of development of the brain. Thus, there is a similarity to the considerable displacement of bulbs found in species belonging to the Cyprinide-type. The peculiar position of the olfactory bulbs in Mugil auratus, however, does not necessarily represent a transitional stage to the Cyprinide-type. The following problems are discussed: (a) the two types of positions of the olfactory bulbs occurring in Teleostomi; (b) whether or not these two types are related only to morphological differences; (c) the possible physiological conditions for the displacement of the olfactory bulbs. It may be assumed that Mugil auratus is well suited for electrophysiological investigations on the action potentials of the primary and secondary olfactory fibers.
\end{abstract}

\section{EINLEITUNG}

Bei den Knochenfischen lassen sich hinsichtlich der Lage der Bulbi olfactorii im Verhältnis zu den Corpora striata zwei Typen, der Salmoniden- und der CyprinidenTyp, unterscheiden (RABL-RÜCKHARD 1882).

Beim S a Imon id en-Ty p mit proximaler Bulbusposition liegen die Bulbi olfactorii dicht an den Corpora striata. Während die sogenannten "Nervi olfactorii“ relativ lang sind, weisen die "Tractus olfactorii" (die Konnektive zwischen Bulbi olfactorii und Corpora striata) nur eine geringe Länge auf (Abb. $1 \mathrm{~A}$ ). Oft sind die Tractus nur histologisch demonstrierbar. Vergleichend anatomisch handelt es sich im übrigen bei den „Nervi olfactorii“" nicht um echte Nerven. Daher sollten sie besser als primäre Tractus olfactorii bezeichnet werden, die Tractus entsprechend als sekundäre Tractus olfactorii (HARDER 1964 u. a.). Diesen Typ findet man bei folgenden Ordnungen: Clupeiformes, Perciformes, Pleuronectiformes, Esociformes, Anguilliformes, Cyprinodontiformes, Macruriformes, Gasterosteiformes, Tetrodontiformes und anderen.

${ }^{1}$ Herrn Prof. Dr. Dr. h. c. W. E. ANkel zum 70. Geburtstag gewidmet. 
Beim Cy priniden-Ty p mit distaler Bulbusposition liegen die Bulbi olfactorii mehr oder weniger dicht an den Geruchsorganen und in deutlichem Abstand von den Corpora striata (Abb. $1 \mathrm{C}$ ). Die primären Tractus olfactorii, jeweils aus mehreren, oft völlig getrennt verlaufenden Bündeln der Fila olfactoria bestehend, sind nur von geringer Länge, die sekundären Tractus haben dagegen eine relativ große Länge: Cypriniformes (mit Ausnahme der Characinoidei), Mormyriformes, Gadiformes, Galaxiformes.

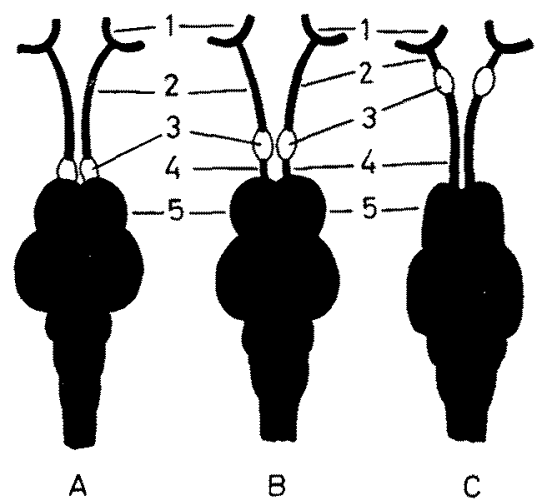

Abb. 1: Schematische Darstellung der Positions Typen des Bulbus olfactorius bei Knochenfischen. (A) proximaler Typ bei Salmo (Typ 1); (B) distanzierte Bulbusposition bei Mugil auratus; (C) distaler Typ bei Urophycis (Typ 2). (1) Geruchsorgan, (2) primärer Tractus olfactorius, (3) Bulbus olfactorius, (4) sekundärer Tractus olfactorius, (5) Corpus striatum

Innerhalb der zweiten Gruppe gibt es bei einigen Gattungen Abweichungen, indem - wie im Falle von Raniceps (Gadidae) sowie Rhodeus und Carassius (Cyprinidae) sowohl primäre als auch sekundäre Tractus olfactorii relativ kurz sind (LISSNER 1923). Hier liegen die Bulbi annähernd in der Mitte zwischen Geruchsorgan und Corpora striata. Man könnte somit von einer intermediären Bulbusposition sprechen. In der ersten Gruppe hingegen waren bisher - wenn man von Uranoscopus absieht, der von der Corpora striata leicht abgesetzte Bulbi olfactorii aufweist (LISSNER 1923) - keine Formen mit deutlich abweichender Bulbusposition bekannt. Bei einer Reihe wenig bekannter Unterordnungen fehlen überhaupt exakte Untersuchungen zur Lage der Bulbi.

\section{BEFUNDE}

Im Rahmen vergleichender Untersuchungen am Vorderhirn verschiedener Knochenfischordnungen konnte nunmehr bei der Goldmeeräsche, Mugil auratus ${ }^{2}$, eine vom proximalen Typus differierende Bulbusposition festgestellt werden: Die Bulbi olfac-

2 Die Tiere wurden während einer Exkursion des I. Zoologischen Instituts der Universität Gießen nach Jugoslawien, und zwar im Litoralbereidk bei Rovinj, gefangen (Juni 1967). 
torii sind hier beträchtlich von den Corpora striata abgerückt. Die bei den Vertretern der beiden anderen Familien (Atherinidae und Sphyraenidae) dieser Ordnung relativ kurzen, sekundären Tractus weisen hier eine bemerkenswerte Länge auf. Dennoch liegen die Bulbi olfactorii bei Mugil auratus wesentlich näher an den Corpora striata als am Geruchsorgan (Abb. $1 \mathrm{~B}$ ). Besonders deutlich entfernt von den Corpora striata erscheinen die Bulbi am lebendfrisch präparierten Gehirn, während die sekundären Tractus durch die Fixierung eine drastische Verkürzung erfahren.

Vergleicht man noch die relative Länge der sekundären Tractus olfactorii bei verschieden großen (langen) Tieren - ein entsprechend verschiedenes Alter wird dabei vorausgesetzt - so ergibt sich folgender Befund: Von vier untersuchten Tieren hatte das offenbar älteste Exemplar (Länge $34,5 \mathrm{~cm}$ ) sekundäre Tractus olfactorii mit der

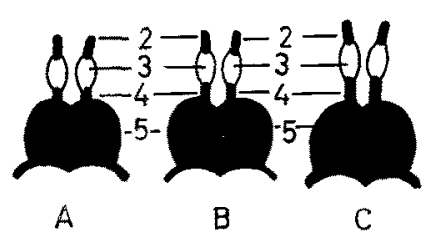

Abb. 2: Position des Bulbus olfactorius bei verschieden alten Tieren von Mugil auratus: (A) bei einem Exemplar von $22,5 \mathrm{~cm}$ Körperlänge, (B) von $26 \mathrm{~cm}$, (C) von $34,5 \mathrm{~cm}$. Zahlen wie in Abbildung 1

relativ größten Länge, das offenbar jüngste Tier (Länge $22,6 \mathrm{~cm}$ ) dagegen hatte die relativ kürzesten Tractus olfactorii (Abb. 2 A bis C). Dabei ist unerheblich, daß auch die primären Tractus olfactorii im Verlaufe des Kopfwachstums eine Verlängerung erfahren. Leider standen keine ein- bis zweijährigen Tiere (Länge unter etwa $20 \mathrm{~cm}$ ) zur Verfügung, und ebenfo fehlte die extreme Längenklasse (über $40 \mathrm{~cm}$ ). Daher kann eine vollständige Aussage nicht gemacht werden. Man darf aber annehmen, daß bei ganz jungen Tieren (einjährig) die Bulbi olfactorii noch annähernd "sitzend" sind, nur durch sehr kurze sekundäre Tractus olfactorii mit den Corpora striata verbunden. Demgegenüber sollten bei sehr großen Tieren (mit über $40 \mathrm{~cm}$ Körperlänge) die Bulbi olfactorii noch weiter von den Corpora striata abgerïckt sein, was zunächst also nur eine Vermutung ist.

Die ventral das Geruchsorgan verlassenden primären Tractus olfactorii, die sich auch bei Mugil auratus aus jeweils zwei Hauptbündeln (bestehend aus zahlreichen kleineren Bündeln) der Fila olfactoria (Axone der Geruchsrezeptoren) zusammensetzen, haben einen rundlichen Querschnitt. Der Querschnitt der wesentlich dünneren sekundären Tractus olfactorii erweist sich dagegen als mehr flach elliptisch. Die Tractus verlaufen noch ein kleines Stück frei, ventral von den Corpora striata, ehe sie in diese eintreten.

Die Bulbi olfactorii weisen eine mittlere Größe auf, wenn man sie etwa vergleicht mit den meist kleinen Bulbi der Percidae und Centrarchidae einerseits und den enorm großen Bulbi der Anguillidae und Verwandten andererseits. 


\section{DISKUSSION}

Unter den Knochenfischen sind bei den verschiedenen Ordnungen die einzelnen Teile des Telencephalon leicht homologisierbar. Das gilt auch für die Bulbi olfactorii, selbst wenn hier - wie eingangs besprochen - entweder eine proximale oder eine distale Position existiert. Sicher ist eine Typisierung des Vorderhirns der Knochenfische nach der Bulbusposition - wie sie RABL-RücKHARDT (1882) vorgeschlagen hat - zunächst nur eine grobe morphologische Lösung, die ich aber durchaus für brauchbar halte. Immerhin erweist sie sich als hilfreich bei der Abgrenzung von bestimmten Ordnungen und Unterordnungen.

Die bei Mugil auratus vorliegende abgerückte Bulbusposition ist ein höchst seltener Fall innerhalb jener Gruppe von Ordnungen, welche die proximale Bulbusposition aufweisen. Wie nun die Untersuchung verschieden alter Tiere gezeigt hat, kommt es hier offenbar während der Spätentwicklung zu einer zunehmenden Distanzierung der Bulbi. Diese ist eine Folge des Längenwachstums der sekundären Tractus olfactorii. Im Gegensatz dazu beginnt das Abrücken der Bulbi bei den Cypriniden (als Vertreter des zweiten Typs), wo sie zunächst ebenfalls dicht am übrigen Vorderhirn liegen, bereits auf früher Entwicklungsstufe. Die Bulbi „wandern“ hier sogar bis dicht an die Nasenkapseln (Sagement 1885). Das gleiche gilt für Polypterus (Goodrich 1928) und ebenso auch für Gadidae (eigene Beobachtungen an Uropbycis, Lota). Entsprechende Angaben über die Verhältnisse bei den Mormyriformes scheinen nicht vorzuliegen. Bei den Formen des ersten Typs - der im übrigen weiter verbreitet ist als der zweite Typ (siehe Einleitung) - persistiert die larvale Situation des dicht anliegenden Bulbus. $\mathrm{Da}$ ein Längenwachstum der sekundären Tractus olfactorii und damit eine Annäherung des Bulbus an das Geruchsorgan unterbleibt, müssen die Fila olfactoria diese bei manchen Arten erhebliche Distanz überbrücken.

Offenbar haben jene Unterschiede in der Bulbusposition bei adulten Tieren aber nichts mit Besonderheiten des Schädelbaues im Zusammenhang mit dem Verlauf der Tractus olfactorii zu tun. So ziehen die primären Tractus olfactorii bei manchen Familien durch die Orbita, bei anderen hingegen nicht (BERg 1958), ohne daß dadurch die Bulbusposition berührt würde. Beispielsweise findet man den ersten Fall innerhalb der Unterordnung Esocoidei (Typ 1) bei den Esocidae, den zweiten bei den Dalliidae und Umbridae. Das gleiche gilt für die Familien der Characinoidei (Typ 2) und anderer Unterordnungen.

Es erhebt sich nun die Frage, inwieweit die bei Mugil auratus existierende distanzierte Bulbusposition als eine Art Obergangssituation zum distalen Typus aufgefaßt werden kann, oder ob die vorliegende $A b$ weichung überhaupt nur einen belanglosen Ausnahmefall darstellt.

Um eine befriedigende Antwort zu erhalten, müßten zunächst weitere Formen vielleicht im Verwandtschaftskreis der Mugiliformes - gefunden werden, die eine ähnliche oder gar noch stärker abweichende Bulbusposition aufweisen als Mugil auratus.

Eine besondere Beachtung sollten aber auch die Cypriniformes erfahren, die hinsichtlich der Bulbusposition eine heterogene Gruppe sind: Bei den Characinoidei (soweit untersucht) liegen die Bulbi dicht an den Corpora striata (eine Ausnahme bilden die Citharinidae), bei den übrigen Unterordnungen dagegen nahe am Geruchsorgan. 
Von den Gadiden weist Raniceps eine "intermediäre" Bulbusposition auf. Diese kommt wahrscheinlich dadurch zustande, daß infolge des frühzeitig beendeten KopfLängenwachstums eine wesentliche Verlängerung der sekundären Tractus olfactorii unterbleibt. Damit handelt es sich jedoch um einen Sonderfall, der ähnlich zu erklären ist wie bei den Cypriniden Carassius und Rbodeus. Im Rahmen der vorliegenden Problematik spielt er keine unmittelbare Rolle.

Primäre Tractus olfactorii von außergewöhnlicher Länge haben Lepisosteus ossens und Verwandte. Dies hängt damit zusammen, daß das Geruchsorgan am rostralen Ende des langausgezogenen Kopfvorderteils liegt, während die Bulbi olfactorii dicht an den Corpora striata "sitzen". Die sekundären Tractus olfactorii sind verschwindend kurz.

Bemerkenswerterweise findet sich auch bei Latimeria die distale Bulbusposition (Mrllot \& ANTHony 1965). Allerdings können keinerlei Aussagen über das frühe Vorderhirn von Latimeria gemacht werden, da bisher Funde von Jungtieren oder gar Larven nicht vorliegen.

Eine gewisse Komplikation für die Beantwortung der angeschnittenen Frage kann im übrigen auftreten, wenn sich herausstellen sollte, daß die in mindestens 5 Ordnungen zu registrierende distale Bulbusposition eine Konvergenzerscheinung ist, was nicht auszuschließen ist; gerade die Verhältnisse bei Latimeria scheinen dafür zu sprechen.

Keineswegs kann man der Auffassung von Lissner (1923) zustimmen, daß die beiden Positionstypen der Bulbi olfactorii nur quantitative Differenzen des Vorderhirns repräsentieren. Wenn dies der Fall wäre, so müßten in allen oder mindestens in

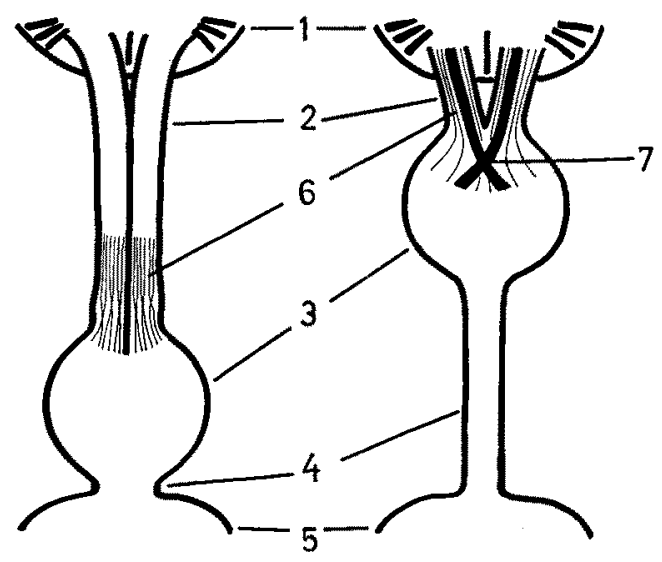

A

$\mathrm{B}$

Abb. 3: Verlauf der Axonbündel (Fila olfactoria) im primären Tractus olfactorius bei Eintritt in den Bulbus olfactorius. (A) Aphbanopus (Typ 1), (B) Ictalurus (Typ 2). (6) Axonbündel, (7) "Chiasma“. Ubrige Zahlen wie in Abbildung 1

einer Reihe von Ordnungen alle möglichen Ubergangsformen existieren. Außerdem sollten dann auch die strukturellen Details der Bulbi olfactorii identisch oder sehr ähnlich sein. Aber weder das eine noch das andere triff $z u$. 
Echte Ubergangsformen - von denen zwar gesprochen wird (Bertin 1954, in Anlehnung an LISSNER 1923) - sind bisher nicht bekannt. Ob nun die Bulbusposition von Mugil auratus als eine solche anzusprechen ist oder ob es sich nur um einen Sonderfall des Typs 1 handelt, kann vorerst noch nicht mit Sicherheit entschieden werden.

Unterschiede in der Struktur des Bulbus olfactorius konnten im Bereich der Eintrittsstelle des primären Tractus olfactorii festgestellt werden. Bei Formen mit distalen Bulbi kommt es dicht hinter der Eintrittsstelle der Tracti in den Bulbus zu einer Uberkreuzung (Abb. 3 B) zweier starker Bündel der Fila olfactoria (Cyprinus: SHeLDon 1912, Ictalurus, Silurus, Synodontis und Pangasius: eigene Befunde). Vertreter des proximalen Bulbus-Typs weisen ein derartig markantes, genau median im rostralen Bulbusbereich liegendes "Chiasma" zweier starker Axonbündel nicht auf (Abb. 3 A). Es finden sich jedoch bei manchen Arten unmittelbar vor der besagten Eintrittsstelle mehr oder weniger zahlreiche Uberkreuzungen kleiner und kleinster Axonbündel (Abb. 3 A) in unregelmäßiger Weise und Verteilung (Osmerus: Holmgren 1920, Salmo, Perca, Micropterus und Aphanopus: eigene Befunde). Über den Verlauf der Axonbündel von Mugil auratus im rostralen Bulbusbereich müssen noch Untersuchungen angestellt werden.

Schließlich muß noch die Frage nach den möglichen Ursachen und Bedingungen für die beim distalen Typ ontogenetisch frühe Distanzierung der Bulbi olfactorii vom Vorderhirn bzw. auch für das relativ späte Abrücken derselben bei Mugil auratus diskutiert werden. Untersuchungen zu diesem Thema fehlen bisher. Einen Hinweis könnten jedoch die Ergebnisse von Giroud, Martinet \& Deluchat (1965) an Embryonen und Jungtieren der Maus liefern, die feststellen konnten, daß nur dann die Bildung des Bulbus olfactorius eingeleitet wird, wenn (vorher) die von den Riechplakoden ausgewachsenen Fila olfactoria das embryonale Vorderhirn erreicht haben. Unterbleibt der Kontakt zwischen den olfactorischen Axonen und dem Vorderhirn, etwa auf Grund von angeborenen Anomalien oder infolge eines experimentellen Eingriffs, so bildet sich der Bulbus olfactorius nicht aus. Groud, Martinet \& Deluchat (1965) schließen daraus auf eine Induktion der Bulbusbildung durch die Fila olfactoria.

Falls es sich nun bei Knochenfischen ähnlich verhält, was noch nicht bewiesen ist, so wäre dann durchaus zu prüfen, ob die Bulbusposition - ganz gleich ob die proximale oder die distale - unter anderem das Ergebnis von Wirkungen ist, die dem "Nervus olfactorius" zuzuschreiben sind. Das Studium der Vorderhirnentwicklung von Mugil auratus sowie auch Ausschaltungsexperimente, könnten zu einer weiteren Aufhellung des Problems beitragen.

Aber nicht nur für vergleichende Arbeiten am Vorderhirn, sondern auch für elektrophysiologische Untersuchungen zur Chemorezeption scheint mir Mugil auratus ein höchst interessantes Objekt. Es lassen sich nämlich unter Umständen Potentialableitungen sowohl von den primären als auch von den sekundären Tractus olfactorii vornehmen, also solche von vor und hinter dem Bulbus (prae- und postbulbär). Die Ableitungen könnten an ein und demselben Tier und möglicherweise - bei entsprechender Versuchsanordnung - sogar simultan durchgeführt werden. Meines Wissens ist dies bisher - wenigstens bei Fischen - nicht gelungen. Bei Arten mit proximalen Bulbi sind die sekundären Tractus, bei solchen mit distalen Bulbi sind die primären Tractus olfactorii zu kurz, um feine Faserbündel oder gar Einzelfasern freilegen zu können. 


\section{ZUSAMMENFASSUNG}

1. Die Position der Bulbi olfactorii der Goldmeeräsche Mugil auratus Russo entspricht grundsätzlich dem sogenannten Salmoniden-Typ (proximale Bulbusposition in Relation zu den Corpora striata), jedoch kommt es während der Spätentwicklung zu einem Abrücken der Bulbi von den Corpora striata.

2. Dieses als "Distanzierung“ bezeichnete Abrücken der Bulbi ist die Folge eines relativ spät einsetzenden Längenwachstums der sekundären Tractus olfactorii.

3. Damit liegt eine Parallele vor zu den Verhältnissen bei Arten, deren Bulbusposition dem Cypriniden-Typ entspricht (distale Position). Keineswegs kann aber in der „distanzierten“ Bulbusposition von Mugil auratus damit schon eine Übergangsform zum Cypriniden-Typ gesehen werden.

4. Das Auftreten der beiden Positionstypen innerhalb der Gruppe der Teleostomi, die Wahrscheinlichkeit qualitativer Unterschiede zwischen den beiden Positionstypen sowie die möglichen physiologischen Voraussetzungen für das Abrücken der Bulbi olfactorii bei den Vertretern des distalen Typs und bei Mugil auratus werden diskutiert.

5. Mugil auratus dürfte auf Grund der distanzierten Position seiner Bulbi besonders geeignet sein für (eventuell simultane) Potentialableitungen von den primären und sekundären olfaktorischen Fasern.

\section{ZITIERTE LITERATUR}

BerG, L. S., 1958. System der rezenten und fossilen Fischartigen und Fische. A. d. Russ. 2. Aufl. VEB Dt. Verl. d. Wiss., Berlin, 310 pp.

Bertin, L., 1954. Système nerveux. In: Traité de zoologie Ed. par P. Grassé. Masson \& Cie, Paris, 13, 855-922.

Giroud, A., Martinet, M. \& Deluchat, C., 1965. Mécanisme de développement du bulbe olfactif. Archs Anat. Histol. Embryal. 48, 205-217.

Goodrich, E., 1928. Polypterus a Palaeoniscid? Palaeobiologica 1, 87-92.

Harder, W., 1964. Anatomie der Fische. T. 1. 2. Schweizerbart, Stuttgart, 1 (T'ext), 308 pp. 2 (Abb.). (Handb. Binnenfisch. Mitteleur. 2 A.)

Holmgren, N., 1920. Zur Anatomie und Histologie des Vorder- und Zwischenhirns der Knochenfische. Acta zool., Stockh. 1, 137-315.

Minlot, J. \& Anthony, J., 1965. Anatomie de Latimeria chalumnae. T. 2. Système nerveux et organes des sens. Centre National de la Recherche Scientifique, Paris, 130 pp.

Lissner, H., 1923. Das Gehirn der Knochenfische. Wiss. Meeresunters. Abt. Helgol. 14, 129-188.

Rabl-Rückhard, H., 1882. Zur Deutung und Entwickelung des Gehirns der Knochenfische. Arch. Anat. Physiol. (Anat. Abt.) Jg, 1882, 111-136.

SAgemenL, M., 1885. Beiträge zur vergleichenden Anatomie der Fische. III. Das Cranium der Characiniden. Morph. Jb. 10, 1-119.

- 1891. Beiträge zur vergleichenden Anatomie der Fische. IV. Das Cranium der Cypriniden. Morph. Jb. 17, 489-595.

Sheldon, R.E., 1912. The olfactory tracts and centers in Teleosts. J.comp. Neurol. 22, 177-346. 